\title{
Control of Supersonic Boundary Layers Using Steady Suction
}

\author{
P.Balakumar* \\ NASA Langley Research Center, Hampton, VA 23681
}

\begin{abstract}
Control of supersonic boundary layers using steady suction through a series of very small two-dimensional strips is numerically investigated at a free stream Mach number of 1.8. The mean flow induced by rows of suction holes is also computed. Both the steady and unsteady solutions are obtained by solving the full NavierStokes equations using the $5^{\text {th }}$-order accurate weighted essentially non-oscillatory (WENO) scheme for space discretization and using third-order total-variationdiminishing (TVD) Runge-Kutta scheme for time integration. Computations for the two-dimensional cases are performed at suction coefficients 0.001 and 0.002 to investigate the stabilizing effects of suction. The simulation showed that a series of shock waves are generated at the slots. The stability results showed that the total amplification is reduced up to the end of the computational domain. However, the growth rates become larger at downstream distances away from the suction region. The computations for the suction holes showed the generation of Mach waves from each hole and the formation of longitudinal vortices.
\end{abstract}

\section{Introduction}

There exist several methods to control laminar boundary layers, e.g. suction, wall cooling, shape modification, artificial blowing. The main purposes of these controls are to: (1) avoid the laminar separation and/or (2) delay or prevent transition from laminar to turbulent flow in laminar boundary layers and, hence, reduce the drag force and/or increase the lift. In this paper, the effects of suction on the stability of supersonic boundary layers are investigated. The effects of suction on a laminar boundary layer are to reduce the thickness of the boundary layer and to make the boundary layer becomes fuller near the wall. Since the viscous instability is directly related to the second derivative of the stream wise velocity at the wall, boundary layers with suction become more stable than those without suction. In flows with adverse pressure gradients, suction removes or weakens the inflection in the velocity profile and, hence, inhibits the inviscid instability.

In theory, with a sufficient amount of suction, it is possible to prevent completely the transition from laminar to turbulent flow. Full chord laminar flows have been obtained at high Reynolds numbers in wind tunnels and in flight using suction. Joslin ${ }^{1}$, in a review article, discusses the limitations of suction in stabilizing the boundary layers. If the suction level exceeds a critical level, the flow becomes turbulent due to instabilities generated near the suction holes ${ }^{2,3}$. This threshold suction level depends on several parameters including the diameter of the suction holes, suction flow rates and alignment of the suction holes. The transition process mainly depends on the boundary layer characteristics and on the frequency, wave number distributions, and amplitudes of the disturbances that enter the boundary layer. The boundary layer profiles depend on the flow parameters such as Mach number, Reynolds number, wall temperature, and the model geometry. The suction modifies the boundary layer profile and makes it more stable.

* Aerospace Engineer, Flow Physics and Control Branch, NASA Langley Research Center, MS 170, AIAA Member.

1 of 16

American Institute of Aeronautics and Astronautics 
When suction is applied through slots or holes, the flow around the suction slots can generate disturbances that can lead to early transition. Another possibility is that external disturbances such as acoustic and turbulence can interact with the flow field near the slots and can enhance the receptivity process.

The objectives of this work are to investigate computationally the flow field induced by the suction slots and holes and to investigate the effects of suction rates on the stability of a supersonic boundary layer over a flat plate at a free stream Mach number of 1.8. Mean flow, stability and receptivity computations are performed for the case where suction is applied through perforated strips. Mean flow computations are also performed for the case where suction is applied through rows of holes. A schematic diagram of the computational set up is depicted in Fig. 1. Recently, an experiment has been performed ${ }^{4}$ to determine the suction-induced tripping of an attachment line boundary layer on 65-degree swept leading edge at Mach number 2.0. The same parameters in this experiment along the attachment line are used in this investigation. Instability waves inside the boundary layer are generated by the interaction of acoustic waves with the boundary layer. In previous studies ${ }^{5,6}$, the interactions of two and three-dimensional acoustic disturbances with and without isolated two-dimensional roughness elements in a supersonic boundary layer have been investigated. The numerical procedure is first to compute the mean flow field with and without suction. The next step is to superimpose acoustic disturbances at the outer boundary of the computational domain and to simulate the evolution of the disturbances inside the boundary layer. A similar procedure is employed in this paper.

\section{Governing Equations.}

The equations solved are the three-dimensional unsteady compressible Navier-Stokes equations in conservation form

$$
\begin{aligned}
& \frac{\partial}{\partial} Q_{i}+\frac{\partial}{\partial x_{j}}\left(F_{j i}-F_{v j i}\right)=0 .
\end{aligned}
$$

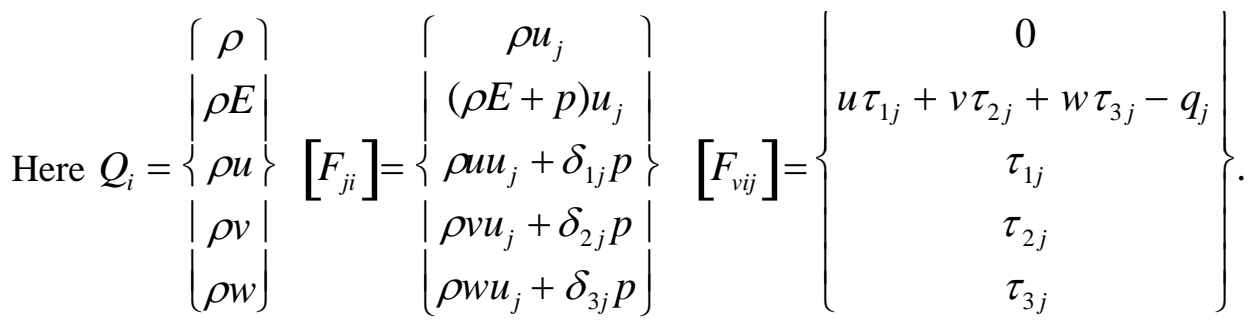

Here $(x, y, z)$ are the Cartesian coordinates, $(u, v, w)$ are the velocity components, $\rho$ is the density, and $p$ is the pressure. $E$ is the total energy given by

$$
\begin{aligned}
& E=e+\frac{u^{2}+v^{2}+w^{2}}{2}, \\
& e=c_{v} T, p=\rho R T .
\end{aligned}
$$

Here $e$ is the internal energy and $T$ is the temperature. The shear stress and the heat flux are given by

$$
\tau_{i j}=\mu\left\{\frac{\partial u_{i}}{\partial x_{j}}+\frac{\partial u_{j}}{\partial x_{i}}-\frac{2}{3} \delta_{i j} \frac{\partial u_{k}}{\partial x_{k}}\right\}, \quad q_{j}=-k \frac{\partial T}{\partial x_{j}} .
$$

The viscosity $(\mu)$ is computed using Sutherland's law and the coefficient of conductivity $(k)$ is given in terms of the Prandtl number $\operatorname{Pr}$. The variables $\rho, p, T$ and velocity are non-dimensionalised by their

$$
2 \text { of } 16
$$

American Institute of Aeronautics and Astronautics 
corresponding reference variables $\rho_{\propto}, p_{\propto}, T_{\propto}$ and $\sqrt{R T_{\infty}}$ respectively. The reference value for length is computed by $\sqrt{1 x_{0} / U_{\infty}}$, where $x_{0}$ is a reference location. For the computation, the equations are transformed from physical coordinate system $(x, y, z)$ to the computational curvilinear coordinate system $(\xi, \eta, \zeta)$ in a conservative manner and the governing equations become

$$
\frac{\partial}{\partial t} \bar{Q}_{i}+\frac{\partial}{\partial x_{j}}\left(\bar{F}_{j i}-\bar{F}_{v j i}\right)=0 .
$$

The components of the flux in the computational domain are related to the flux in the Cartesian domain by

$$
\bar{Q}_{i}=\frac{Q_{i}}{J},\left[\bar{F}_{j i}\right]=\frac{J}{|J|}\left[F_{j i}\right]
$$

$$
\text { where } J=\left\lceil\frac{\partial(\xi, \eta, \zeta)}{\partial(x, y, z)}\right\rceil \text {. }
$$

\section{Solution Algorithm}

The governing equations are solved using a 5th order accurate WENO scheme for space discretization and using a third order, total variation diminishing (TVD) Runge-Kutta scheme for time integration. These methods are suitable in flows with discontinuities or high gradient regions. These schemes solve the governing equations discretely in a uniform structured computational domain in which flow properties are known point wise at the grid nodes. They approximate the spatial derivatives in a given direction to a higher order at the nodes, using the neighboring nodal values in that direction, and they integrate the resulting equations in time to get the point values as a function of time. Since the spatial derivatives are independent of the coordinate directions, the method can easily add multidimensions. It is well known that approximating a discontinuous function by a higher order (two or more) polynomial generally introduces oscillatory behavior near the discontinuity, and this oscillation increases with the order of the approximation. The essentially nonoscillatory (ENO) and the improvement of these WENO methods are developed to keep the higher order approximations in the smooth regions and to eliminate or suppress the oscillatory behavior near the discontinuities. They are achieved by systematically adopting or selecting the stencils based on the smoothness of the function, which is being approximated. Ref. 7 explains the WENO and the TVD methods and the formulas and Ref. 8 gives the application of the ENO method to the N-S equations. Ref. 9 describes in detail the solution method implemented in this computation.

At the outflow boundary, characteristic boundary conditions are used. At the wall, the simulation uses viscous conditions for the velocities and a constant temperature condition, and it computes density from the continuity equation. In the spanwise direction, symmetric and periodic conditions are used at the boundaries. In the mean flow computations, the simulation prescribes the free-stream values at the upper boundary, which lies outside the bow shock. In the unsteady computations, it superimposes the acoustic perturbations to the uniform mean flow at the upper boundary. The procedure is to first compute the steady mean flow by performing unsteady computations using a variable time step until the maximum residual reaches a small value $\sim 10^{-11}$. These computations use a CFL number of 0.3 . The next step is to introduce unsteady disturbances at the upper boundary of the computational domain and to perform time accurate computations to investigate the interaction and evolution of these disturbances downstream.

In the suction case, boundary conditions are applied on the surface across the suction strips. Across each suction slot a suction distribution of the form

3 of 16

American Institute of Aeronautics and Astronautics 


$$
(\rho v)_{n}=f_{\max } \sin ^{2}\left(\frac{\pi\left(x-x_{\text {start }}\right)}{\left(x_{\text {end }}-x_{\text {start }}\right)}\right)
$$

is used to get the required mass flow rate. Here $f_{\max }$ is the maximum amplitude of the suction distribution selected to match the total suction rate and $x_{\text {start }}$ and $x_{\text {end }}$ are the beginning and end of each suction slot. The other flow quantities are obtained using the characteristic boundary conditions described in Ref. 10.

The symmetric acoustic field that impinges on the outer boundary is taken to be in the following form.

$$
\begin{aligned}
p_{a c}^{\prime}= & \operatorname{Real}\left\{\tilde{p}_{a c} e^{i \alpha_{a c} x \pm i \beta z+i \varepsilon_{a c} y-i \omega t}\right\} \\
& +\operatorname{Real}\left\{\tilde{p}_{a c} e^{i \alpha_{a c} x \pm i \beta z-i \varepsilon_{a c} y-i \omega t}\right\} .
\end{aligned}
$$

Here $\alpha_{a c}, \beta_{a c}, \varepsilon_{a c}$ are the acoustic wave numbers, and $\omega$ is the frequency of the acoustic disturbance. The wavenumber in the $y$-direction $\varepsilon_{a c}$ determines the incident angle of the acoustic waves and in this paper computations are performed for zero incident angle, $\varepsilon_{a c}=0.0$.

\section{Results}

Computations are performed for a supersonic flow over a semi-infinite flat plate with a blunt leading edge. Table 1 gives the flow parameters and Fig. 1 shows the schematic diagram of the computational set up. The leading edge of the plate is modeled as a super ellipse of the form

$$
\frac{(x-a)^{4}}{a^{4}}+\frac{y^{2}}{b^{2}}=1 \text {. }
$$

Here $b$ is the thickness of the plate and is 0.001 inches. The aspect ratio $a / b$ is taken as 10 hence the blunt leading edge is joined with the straight portion of the plate at $x=0.01$ inch.

In the first part, the suction is applied through perforated strips, which extend from 0.55 in. to 4.55 inch. The width of the strip is $d=0.02 \mathrm{in}$. and the spacing between the strips $s=5^{*} d=0.10$ inches. The number of suction strips are $N=40$. The maximum mass flow rate $f_{\max }$ in eq.(7) is obtained from the equation

$$
f_{\max }=2 q \frac{s}{d} \text {. }
$$

Here $\mathrm{q}$ is the suction coefficient per unit area given by

$$
q=\frac{\text { total suction }}{\rho_{\infty} U_{\infty} A} \text {. }
$$

Computations are performed for suction rates of $q=0.001$ and 0.002 .

\section{Table 1 Flow parameters for the wind tunnel model}

Free stream Mach number: $M_{\propto}=1.81$

Free stream Reynolds number: $R e_{\propto}=2.18 * 10^{6} / \mathrm{ft}$

Free stream density: $\rho_{\propto}=1.0996 * 10^{-2} \mathrm{lbm} / \mathrm{ft}^{3}$

Free stream pressure: $p_{\propto}=184.03 \mathrm{lbf} / \mathrm{ft}^{2}$ 
Free stream velocity: $U_{\propto}=1573.81 \mathrm{ft} / \mathrm{s}$

Free stream temperature: $T_{\propto}=313.70{ }^{\circ} \mathrm{R}$

Free stream kinematic viscosity: $v_{\infty}=1.7882 * 10^{-4} \mathrm{ft}^{2} / \mathrm{s}$

Wall temperature: $=544.67^{\circ} \mathrm{R}$

Prandtl number: $\operatorname{Pr}=0.72$

Ratio of specific heats: $\gamma=1.4$

Length scale $\sqrt{\frac{v_{\infty} x_{0}}{U_{\infty}}}=1.9526 * 10^{-4} \mathrm{ft}$. ( $x_{0}=1.0 \mathrm{in}$.)

The boundary layer thickness at $\mathrm{x}=1$ in.: $\delta_{0}=.025$ in.

Non-dimensional frequency $F=1 * 10^{-5}$ is equivalent to $41.0 \mathrm{kHz}$

The non-dimensional frequency $F$ is defined as $F=\frac{2 \pi \nu_{\infty} f}{U_{\infty}^{2}}$,

where $f$ is the frequency in Hertz.

The grid is generated using analytical formulae. The grid stretches in the $\eta$ direction close to the wall and is uniform outside the boundary layer. In the $\xi$ direction, the grid is symmetric about the leading edge and very fine near the nose and is uniform in the flat region. The grid is uniform in the spanwise direction. The outer boundary that lays outside of the shock follows a circle near the nose region with its vertex located a short distance upstream of the nose and follows a parabola downstream of the nose to capture the boundary layer accurately. The computational domain extends from $x=-0.015$ to 18.0 inches in the axial direction. Calculations were performed using a grid size $(9001 * 251 * 11)$. Due to the very fine grid requirement the computations become very expensive to simulate in the entire domain at once. To overcome this, calculations are performed in two steps. First, the computations are done in zones. Second, the flow properties in the middle of previous zone are fed as inflow conditions for the second larger domain and the computations are carried out.

\section{Mean flow.}

Figure 2 shows the mean flow v-velocity contours without suction computed using the WENO code. Figure 2a shows the entire domain and Fig. 2b shows the flow field near the nose region. Figures 3(a-d) show the v-velocity contours and the streamlines for the suction case $q=0.002$. Figure 3(a) depicts the contours for the entire domain and Fig. 3(b) shows the flow field up to the end of the suction. Figure 3(c) shows the results across several suction strips and Fig. 3(d) shows the streamlines near one suction slot. The boundary layer thickness at $\mathrm{x}=2.75 \mathrm{in}$. is about 0.025 inches. The figures clearly show the Mach waves that originated from the suction strips. This has been observed in several experiments ${ }^{11}$. The streamline patterns show that very small, about $7 \%$ of the boundary layer is sucked inside by the suction. At the end of the suction slot about $10 \%$ of the boundary layer is sucked into the slot. The flow is entering the slot approximately 10 degrees to the horizontal. This suggests that the suction plenum could be aligned along this direction to avoid flow separation at the lip. The contour field in Fig. 3(c) shows the negative and positive $v$ velocities across the slot, which extends from 2.74 to 2.76 inches. This shows that the streamlines are coming towards the slot from upstream and turn back before turning down towards the next slot. 
Figures 4(a-b) show the pressure variations along the surface across the suction region. Figure 4(b) shows the distribution of pressure and the suction velocity across three slots. There is a sharp increase in pressure across each slot. The increase is from 0.86 to 1.06. Between two slots the pressure variation is smooth. Figures 4(c-d) show the Mach number variation along the edge of the boundary layer above the suction region. Figure 4(d) shows the expanded view across three suction slots. There is a sharp decrease in Mach number across each suction slot. The decrease is from 1.90 to 1.75. This has also been observed in experiments ${ }^{11}$. Figure 5 shows the density and u-velocity profiles at different axial locations $\mathrm{x}=3,8$ and 16 in. with and without suction. The location $\mathrm{x}=3$ is located between two suction slots and $\mathrm{x}=8$ and 16 inches are located downstream of the suction region. As expected the boundary layer becomes thinner and fuller with suction and this will lead to stabilization of the boundary layer. Increasing distances from the suction, the boundary layers with suction approaches the profiles without suction.

\section{Linear stability}

After the steady mean flow is calculated, linear stability analysis is performed. Figures 6 and 7 show the growth rates and N-Factor curves for different non-dimensional frequencies and spanwise wave numbers. First observation is that with suction there are no unstable disturbances upstream of $\mathrm{x}=4.5$ inches with the suction. All the disturbances are stabilized up to $\mathrm{x}=5.5$ inches. The N-Factor diagram shows that up to the computational domain of $\mathrm{x}=18$ inches, the total amplification is less with suction. The maximum N-Factors are about 5.0 without suction and about 3.6 with the suction. However, the growth rate curves show an interesting behavior. The growth rate curve for a fixed frequency is smaller up to a certain distance from the end of the suction and it becomes larger downstream. Hence to stabilize the growth rate completely, the suction has to be applied continuously for a longer distance. If the objective is to laminarize the boundary layer based on total amplifications, optimum suction distribution could be found.

\section{Interaction of three-dimensional acoustic waves with the boundary layer.}

After the mean flow is obtained, three-dimensional slow acoustic disturbances are superimposed at the outer boundaries and time accurate simulations are performed to investigate the interaction of acoustic waves and the boundary layers with and without suction. The non-dimensional frequencies and the spanwise wave numbers are $F=0.75^{*} 10^{-5}, \beta=0.050$ and $F=1.50 * 10^{-5}, \beta=0.025$. In the first case the boundary layer without suction is almost neutral and the second case gives the largest $\mathrm{N}$-factor close to the end of the computational domain. To remain in the linear regime, small initial amplitude of $\tilde{p}_{a c} / p_{\infty}=1.0 * 10^{-5}$ is prescribed for the free-stream acoustic waves.

Figure 8(a) shows the results for the evolution of the unsteady fluctuations with the suction above the suction slots obtained from the simulation at a fixed time. Near the surface, the interactions of the acoustic disturbances with the shock waves originating from the suction slots are clearly seen. The wavelength of the acoustic disturbances is about 0.40 inches and the wavelength of the slots is 0.10 inches. Multiple shocks are interacting with one acoustic wave. Figure 8(b) shows the pressure perturbations along the surface for the cases with and without suction. Without the suction the disturbances continually decay. With the suction the larger disturbances are excited above the suction slots and remain almost the same across the suction region. However, they become almost the same magnitude further downstream as for the case without suction.

Figure 9 shows the amplitude of the pressure fluctuations along the wall for the second higher frequency case. This wave is amplified for both cases with and without suction. The figure clearly shows the initial generation and the eventual exponential growth of the instability waves inside the boundary layer for the case without suction. As predicted from the linear theory, the disturbances start to grow exponentially from $X \sim 2.75$ in. for the case without suction. However, as expected for the case with the suction the disturbances are decaying up to $\mathrm{x} \sim 6.0$ inches.

6 of 16

American Institute of Aeronautics and Astronautics 


\section{Mean flow induced by rows of suction holes.}

Computations are performed to solve for the mean flow field induced by rows of suction holes at a small suction coefficient of 0.0005 . At higher suction rates, the shocks that originated from the suction holes are oscillating and the solution did not converge. The diameters of the holes are $0.02 \mathrm{in}$. and the spacing between the holes in the streamwise and in the spanwise directions are 0.10 inches. The suction holes extend from 0.55 inches to 4.55 inches in the streamwise direction. There are 40 holes in the streamwise direction and symmetric conditions are employed in the spanwise direction. The boundary conditions are applied on the surface across the suction holes. Across each suction hole a suction distribution of the form

$$
(\rho v)_{n}=f_{\max } \cos ^{2}\left(\frac{\pi\left(r-r_{0}\right)}{d}\right)
$$

is used to get the required mass flow rate. Here $f_{\max }$ is the maximum amplitude of the suction distribution selected to match the total suction rate and $r_{0}$ is the center of the hole and $\mathrm{d}$ is the diameter of the hole. The other flow quantities are obtained using the characteristic boundary conditions described in Ref. 9.

Figures 10(a-c) show the v-velocity contours and the streamlines. Figure 10(b) shows the results across several suction holes and Fig. 10(c) shows the streamlines near one suction hole. The boundary layer thickness at $\mathrm{x}=1.75 \mathrm{in}$. is about 0.015 inches. The figures clearly show the Mach waves that originated from the suction holes. The streamline patterns show that about $6 \%$ of the boundary layer is sucked inside by the suction. The flow is entering the suction hole approximately 10 degrees to the horizontal in the symmetry plane. The contour field in Fig. 10(b) shows the negative and positive $\mathrm{v}$ velocities across the slot, which extends from 1.64 to 1.66 inches. This shows that the streamlines are coming towards the slot from upstream and turn back before turning down towards the next slot.

Figures 11(a-b) show the pressure variations along the surface across the suction region. Figure 11(b) shows the distribution of pressure and the suction velocity across three slots. As observed in the suction slot case, there is a sharp increase in pressure across each hole. The increase is from 0.86 to 1.16. Between two slots the pressure variation is smooth. Figure 12 depicts the contours of the u-velocity in the plan view, $\mathrm{X}-\mathrm{Z}$ plane, at a height of $1 / 4$ boundary layer. The contours consist of high velocity legs on either side of the low velocity center region. This resembles the flow behind three-dimensional roughness elements in a boundary layer. Figure 13 shows the contours of the axial vorticity in the cross planes, Z-Y, at different axial stations $\mathrm{x}=1.1,1.6$ and 2.4 inches. The figure also includes the streamlines in this plane plotted using $(\mathrm{w}, \mathrm{v})$ velocity components. It is seen that longitudinal counter rotating vortices are generated on either side of the suction holes. These vortices become bigger and move up with increasing distance downstream.

\section{Discussion and Conclusions}

Control of supersonic boundary layers using steady suction through a series of very small twodimensional strips is numerically investigated at a free stream Mach number of 1.8. The instability waves are generated by the acoustic disturbances, which are superimposed at the outer boundary of the domain. Simulations are performed with and without suctions. Computations are performed for suction coefficients 0.001 and 0.002 and most of the results are presented. The suction is applied through forty fine slots of 0.02 inches width and 0.10 inches spacing. The suction extends from 0.5 to 4.5 inches. Computations are also performed to solve for the mean flow induced by rows suction holes for a small suction coefficient of 0.0005 . 
The mean flow computations showed that shocks are generated at each slot with the sharp increase in pressure across the slot. The pressure varies smoothly between the slots. Associated with this shock is a decrease in Mach number across the slot at the edge of the boundary layer. The pattern of the streamlines near the slot shows that the fluid enters the slot at an angle of 10 degrees to the horizontal. The boundary layer profiles become thinner by the suction and the effect is present up to the end of the computational domain.

The stability calculations show that the boundary layer is completely stabilized over the suction region. The boundary layer starts to become unstable downstream of the suction. However, the growth rates remain lower than that without the suction for a long distance downstream. This distance depends on the amount of suction applied. Further downstream, the growth rates become larger than that without suction. However, the total amplification is still less than that without suction. The implication is that to have complete stabilization, the suction should be applied for the entire domain of interest. If the goal is to have a low N-Factors in the domain of interest the suction should be applied to a region near the neutral point.

The simulation with the suction holes shows the steep variations of the pressure and the Mach wave across each hole. The formation of longitudinal vortices is also observed. At a higher suction level, the shocks that are generated near the holes are oscillating and the solution did not converge. Whether it is numerical or is physical has not been resolved yet.

\section{References}

1. Joslin, Ronald D., “Overview of Laminar Flow Control,” NASA/TP-1998-208705, NASA, Langley Research Center, Hampton, VA.

2. Goldsmith, J., 1957, " Critical Laminar Suction Parameters for Suction into an Isolated Hole or a Single Row of Holes,” Northrop Aircraft Report No. BLC-95.

3. Meitz, H. L., and Fasel, H. F., "Navier-Stokes Simulations of the Effects of Suction Holes on a Flat Plate Boundary Layer,” Application of Direct and Large Eddy Simulation to Transition and Turbulence, AGARD-CP551. 1994.

4. Powel, A. G., Beeler, G. B., and King, R. A., “Attachment-line Tripping due to Suction Through a Micro-perforated Skin at Mach 2”, AIAA Paper 2006-3589, 2006.

5. P. Balakumar, "Transition in a Supersonic Boundary layer Due to Roughness and Acoustic Disturbances,” AIAA Paper 2003-3589, 2003.

6. P. Balakumar, "Transition in a Supersonic Boundary layer Due to Acoustic Disturbances," AIAA Paper 2005-0096, 2005.

7. Shu, Chi-Wang, “ Essentially Non-Oscillatory and Weighted Essentially Non-Oscillatory Schemes for Hyperbolic Conservation Laws,” NASA/CR-97-206253 and ICASE Report N0. 976

8. Atkins, H. L., "High-Order ENO Methods for the Unsteady Compressible Navier-Stokes Equations,” AIAA Paper 91-1557, 1991.

9. Balakumar, P., Zhao, H., and Atkins, H., "Stability of Hypersonic Boundary_layers Over a Compression Corner,” AIAA Paper 2002-2848, 2002.

10. Sesterhenn, J., "A Characteristic-type Formulation of the Navier-Stokes Equations for High Order Upwind Schemes,” Computers \& Fluids, 30, pp. 37-67, 2001.

11. Groth, E. E., “Boundary Layer Suction Experiments at Supersonic Speeds”. Boundary Layer and Flow Control. Volume 2, G. V. Lachmann, ed., Pergamon Press, pp. 1049-1076. 1961.

8 of 16

American Institute of Aeronautics and Astronautics 


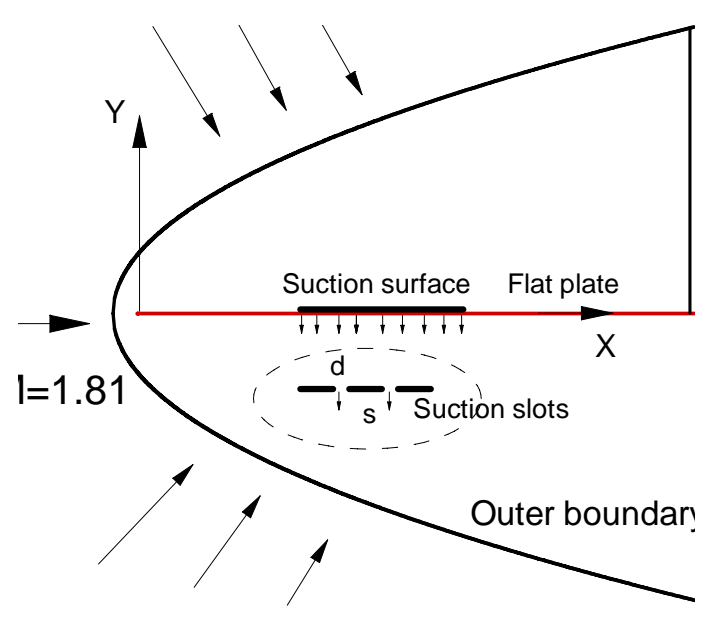

$\mathrm{M}_{\infty}=1.81$

$\operatorname{Re}=2.18 * 10^{6} / \mathrm{ft}$

$\mathrm{T}_{\infty}=313.7^{\circ} \mathrm{R}$

Leading edge thickness $2 b=.002$ in.

Suction is applied from $x=0.55$ to 4.55 in.

Leading edge

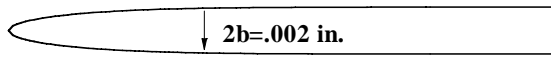

Figure 1. Schematic diagram of the computational model.

(a)

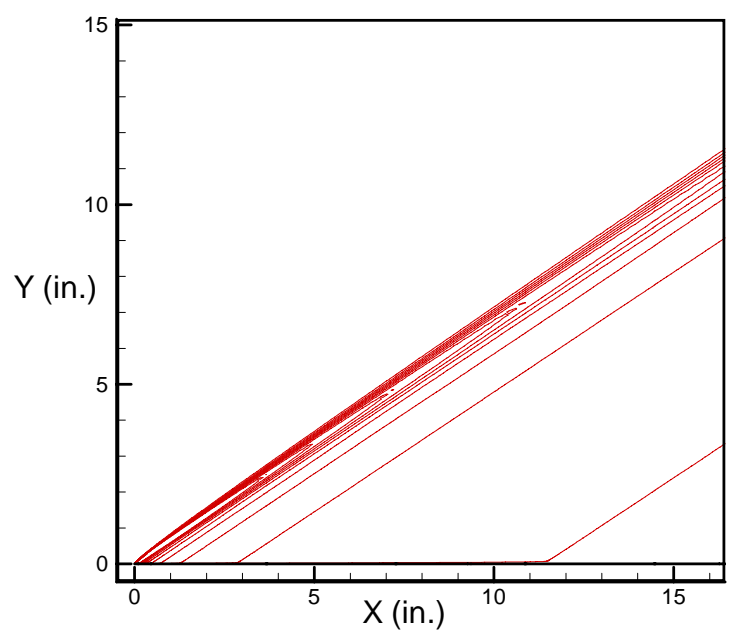

(b)

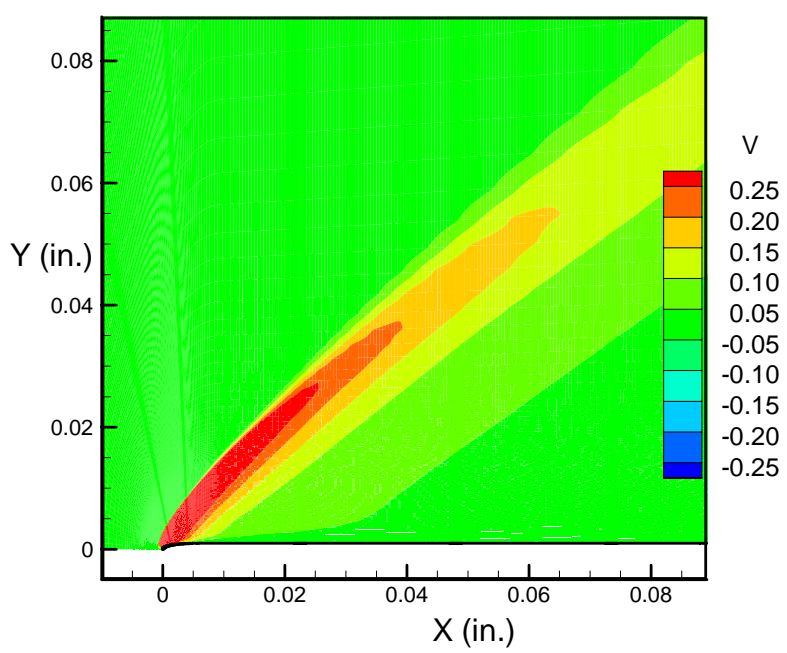

Figure 2. Contours of the v-velocity for flow over a flat plate with a blunted leading edge at $M=1.8$ without suction. 
(a)

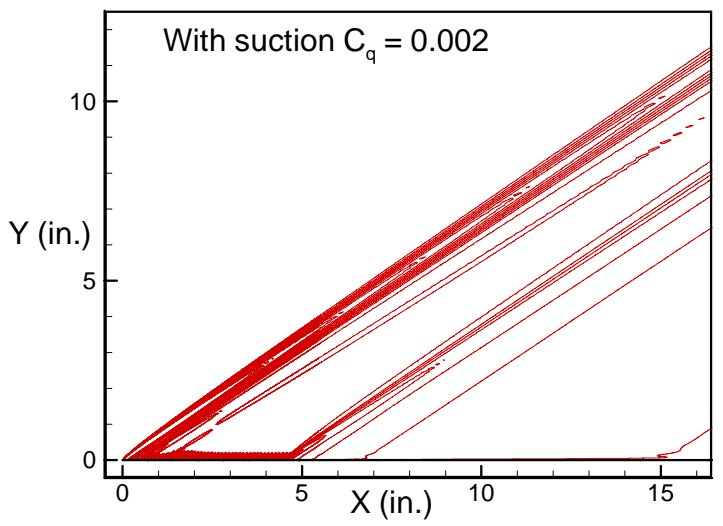

(c)

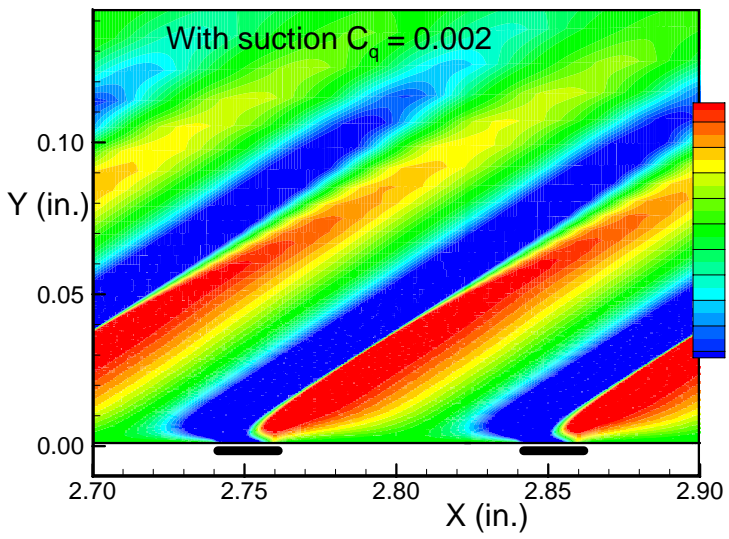

(b)

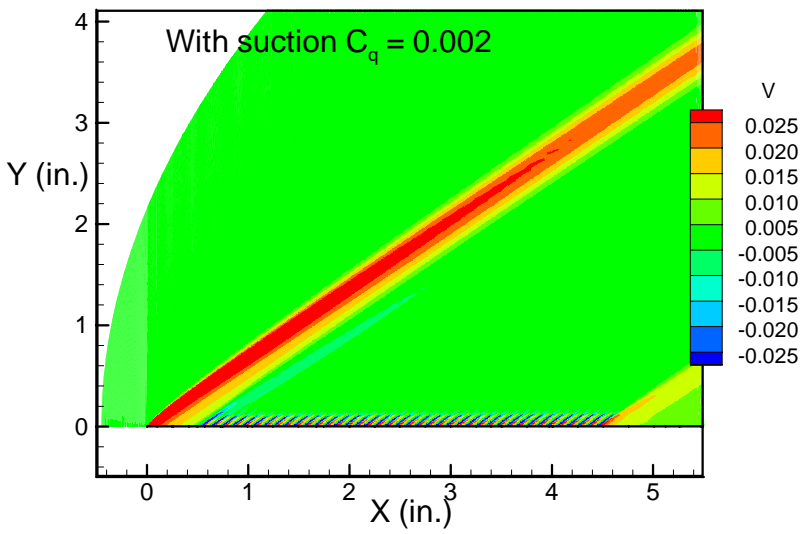

(d)

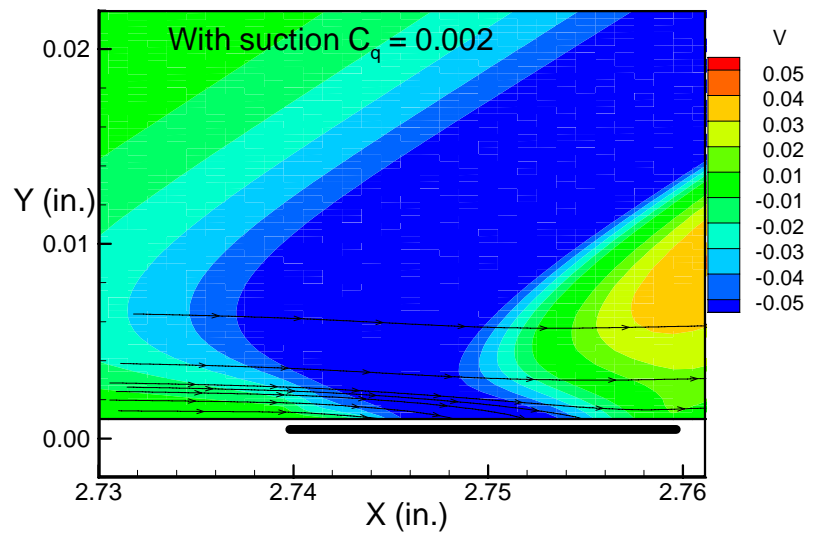

Figure 3. Contours of the v-velocity for flow over a flat plate with a blunted leading edge at $M=1.8$ with suction. 
(a)

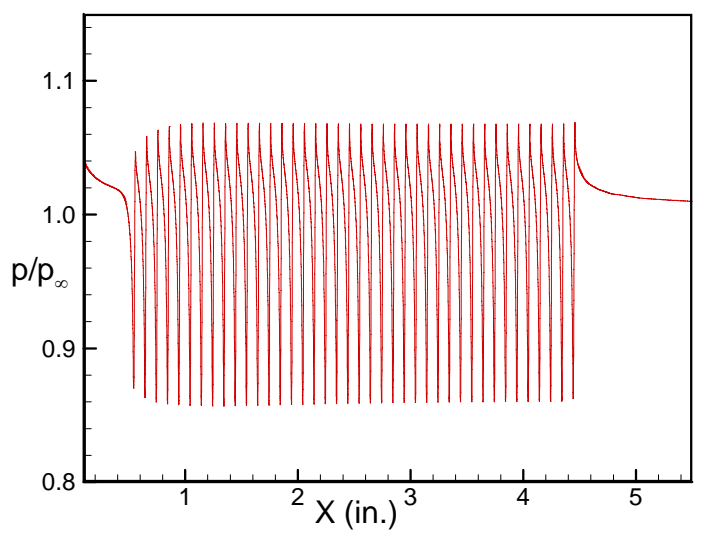

(c)

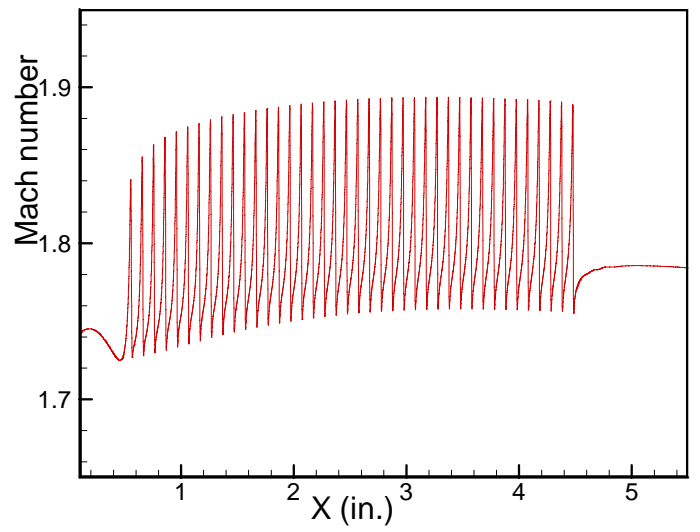

(b)

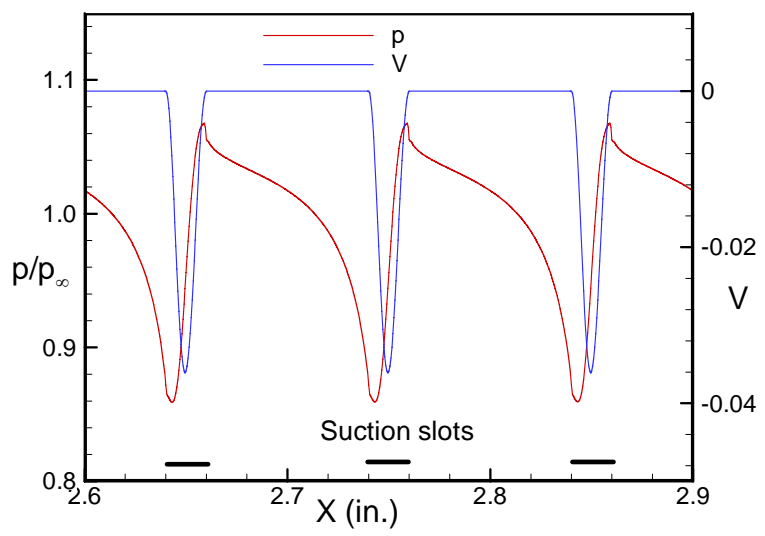

(d)

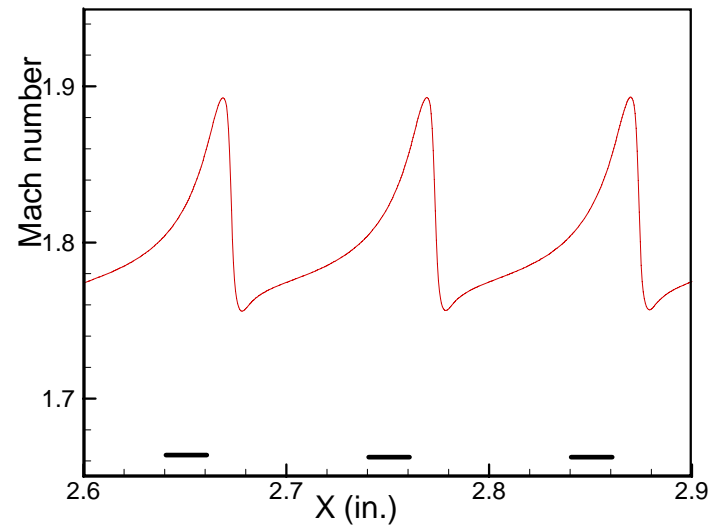

Figure 4. Pressure variation along the surface (a) and (b) with the suction $C_{q}$. Mach number variation along the edge of the boundary layer (c) and (d). 
(a)

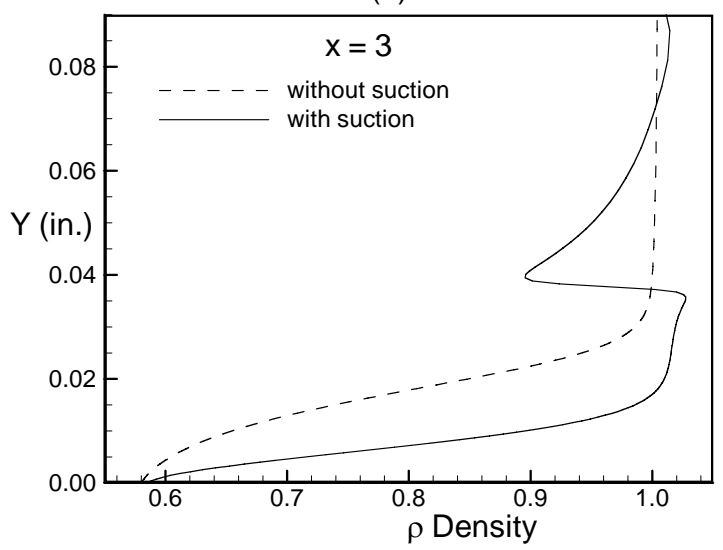

(c)

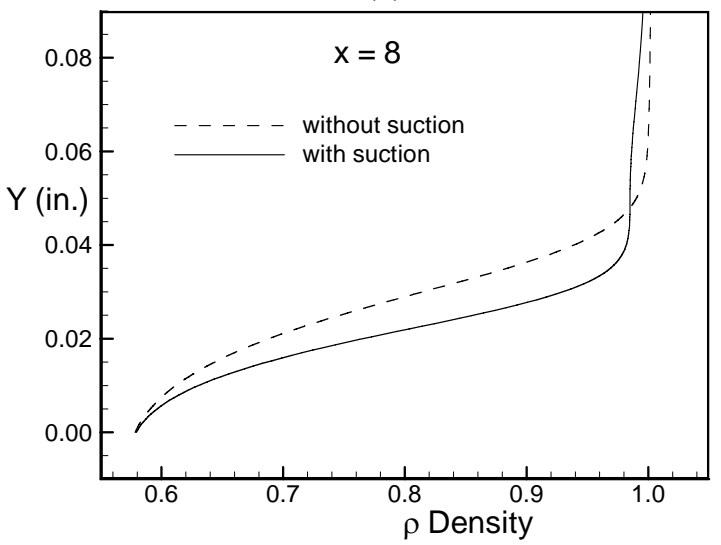

(b)

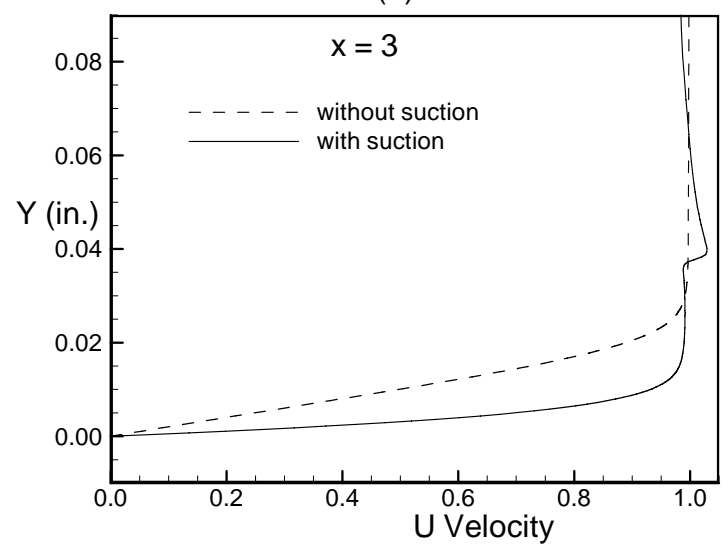

(d)

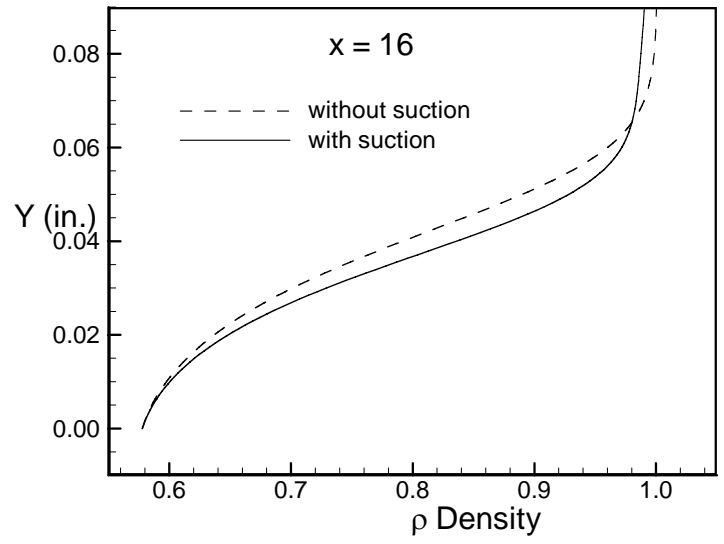

Figure 5. Mean density and $U$ velocity profiles at $x=3,8,16$ in. with and without suction.

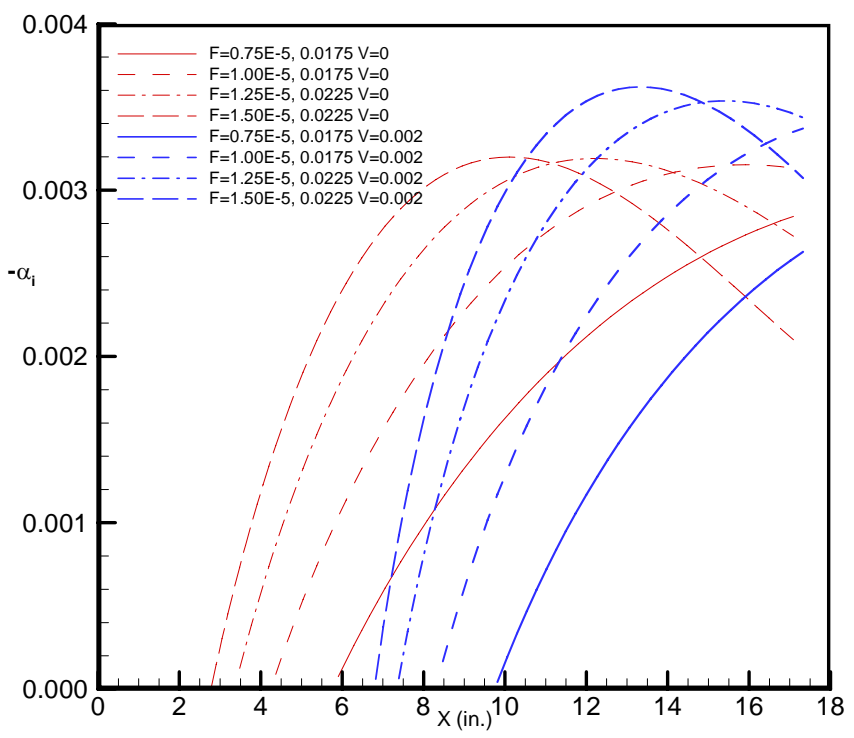

Figure 6. Growth rate computed from linear stability with and without suction.

12 of 16

American Institute of Aeronautics and Astronautics 


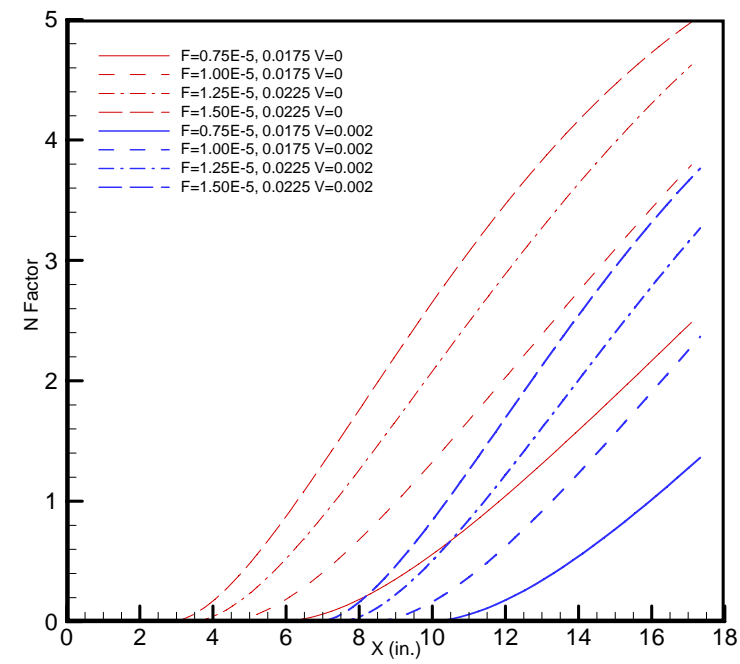

Figure 7. N-Factors with and without suction

(a)

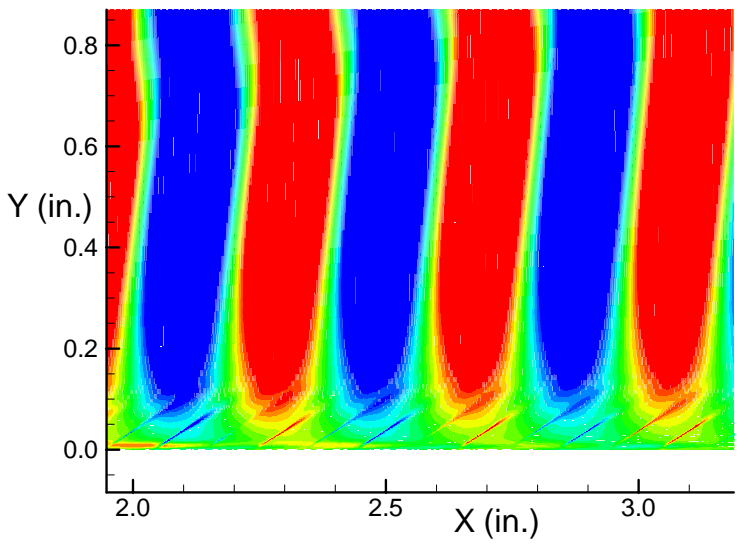

(b)

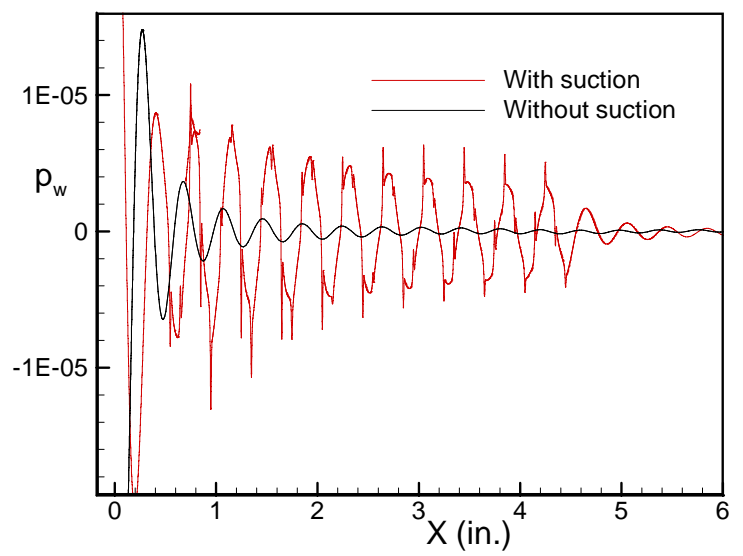

Figure 8. Density contours near the suction slots (a) and the pressure disturbances along the surface for the cases with and without suction. $F=0.75 E-5, \beta=0.05$. 


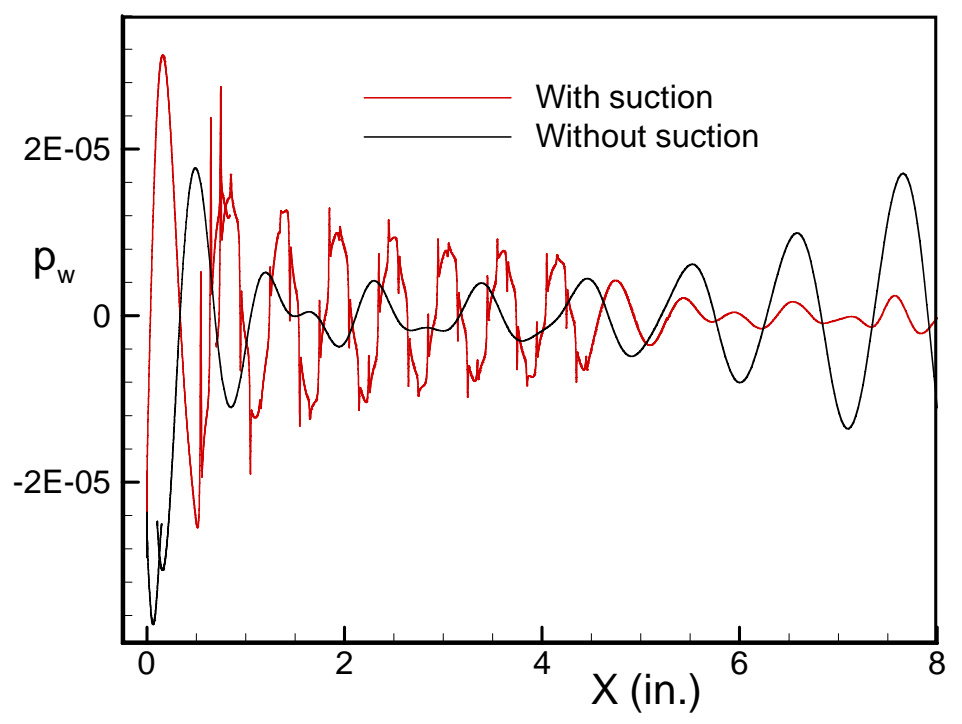

Figure 9. The pressure disturbances along the surface for the cases with and without suction. $\mathrm{F}=$ 1.5E-5, $\beta=0.025$.

(a)

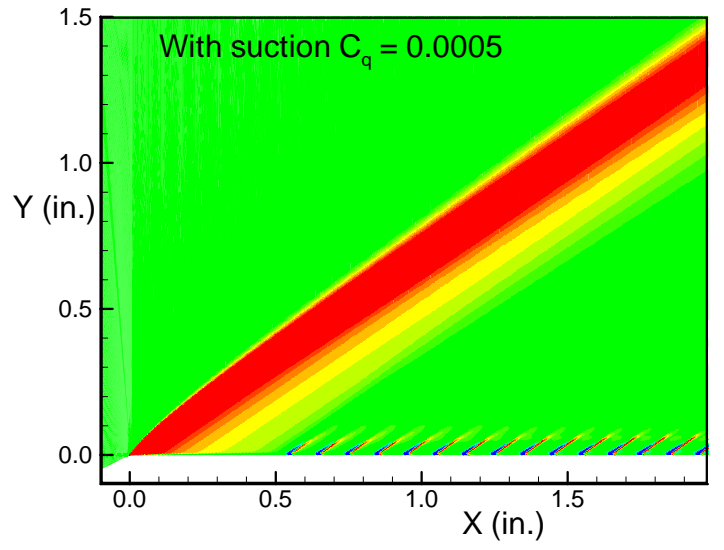

(b)

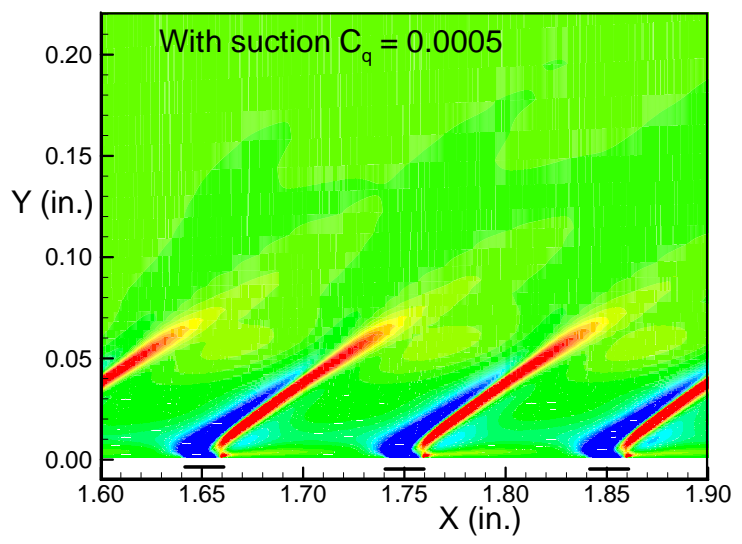

(c)

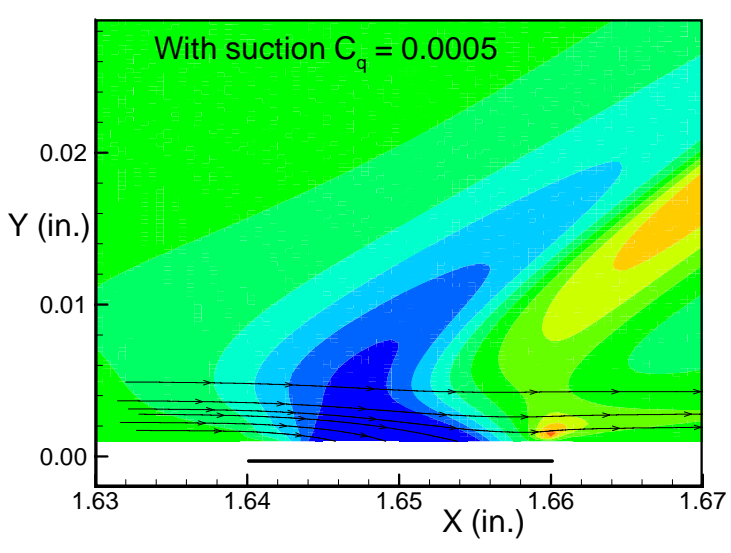

Figure 10. Contours of the v-velocity with suction applied through holes. 
(a)

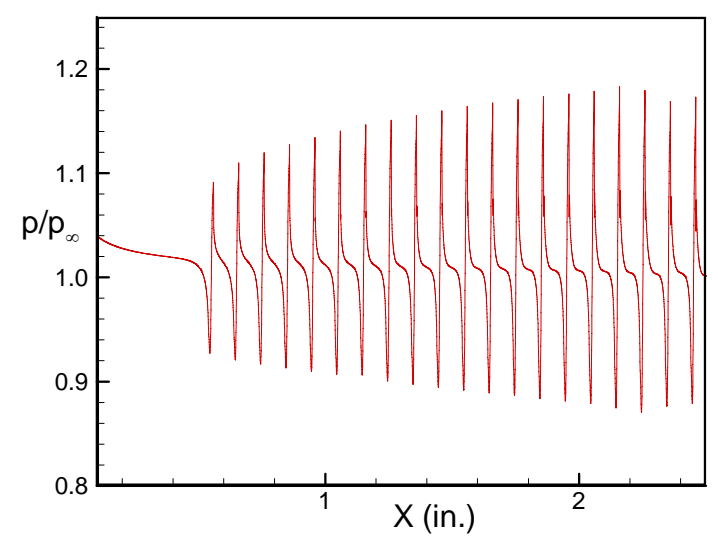

(b)

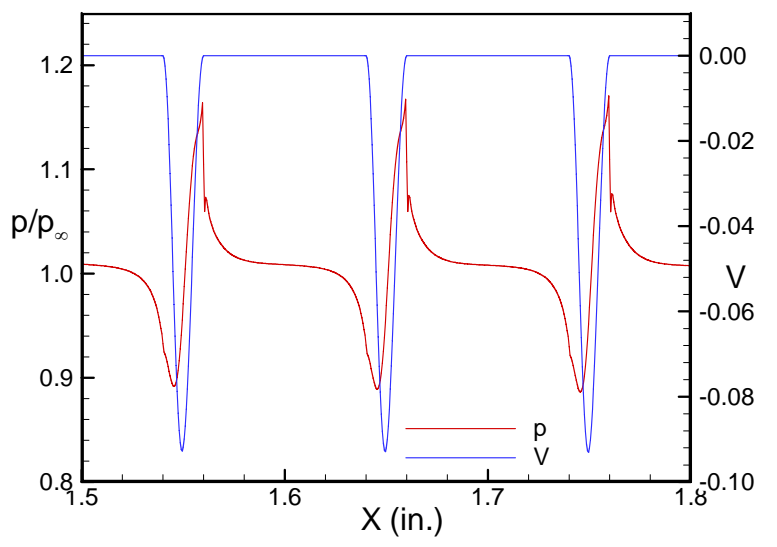

Figure 11. Pressure variation along the surface (a) and the suction velocity (b) with the suction applied through holes.

(a)

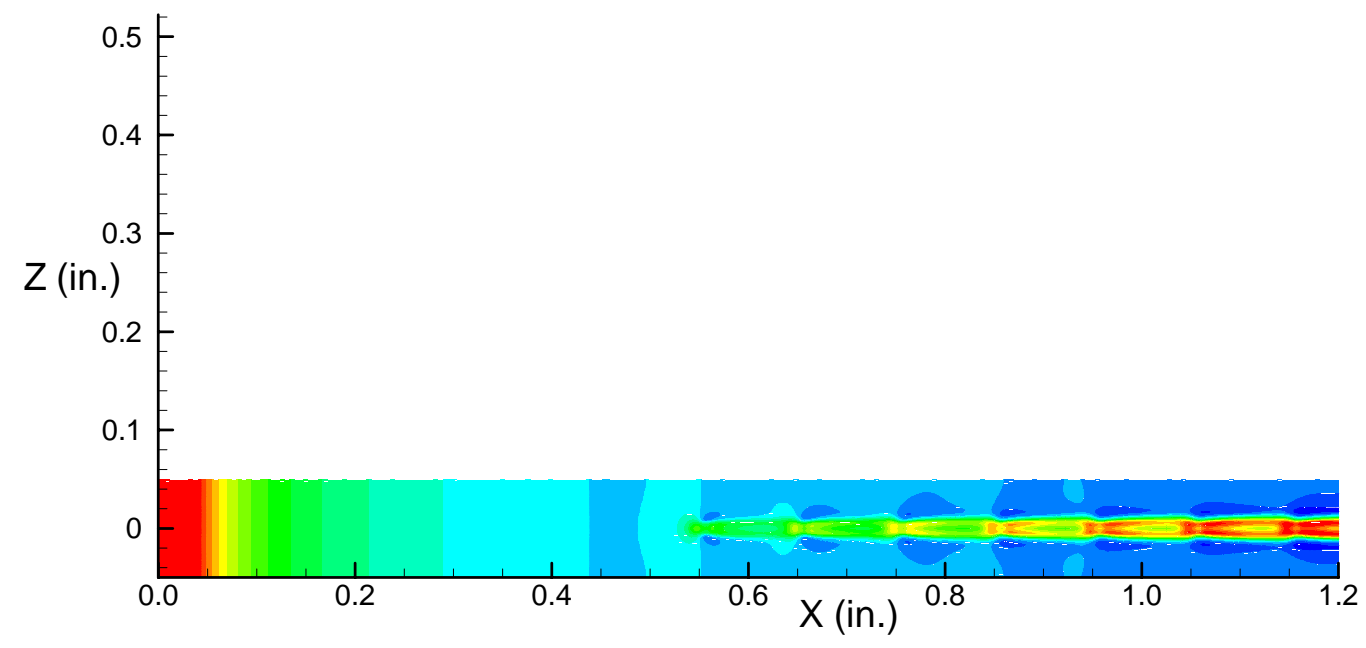

(b)

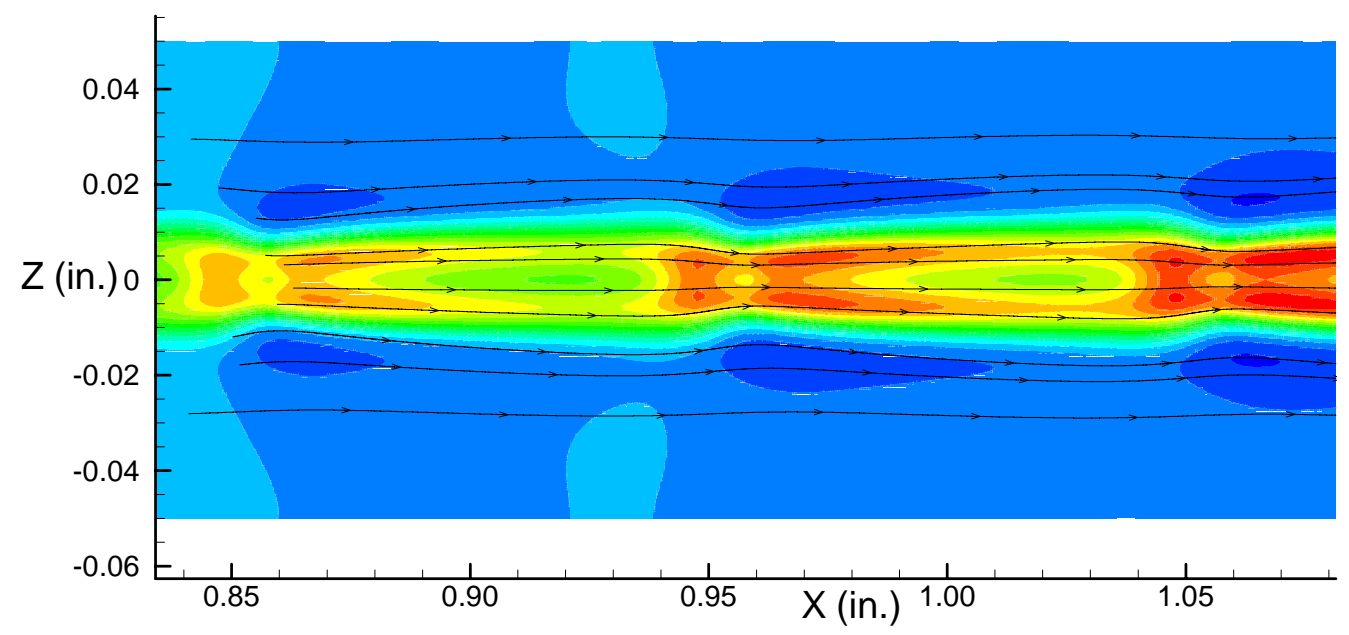

15 of 16

American Institute of Aeronautics and Astronautics 
Figure 12. Contours of $U$ velocity in $X-Z$ plane at a constant $Y$.
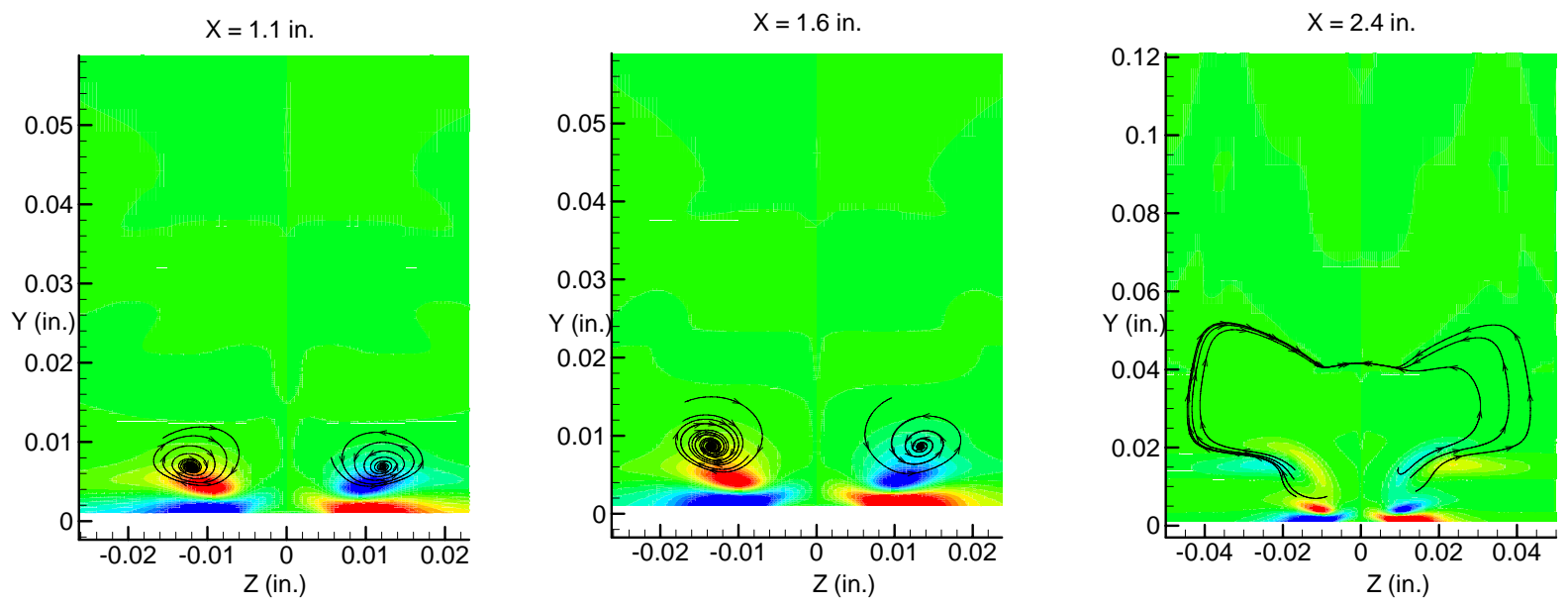

Figure 13. Contours of axial vorticity in $\mathrm{Z}-\mathrm{Y}$ planes at different $\mathrm{X}$ stations 1.1, 1.6, 2.4 inches. 